\title{
All-optical Manipulation of Photonic Membranes
}

\author{
Blair C. Kirkpatrick ${ }^{a}$, Martin Ploschner $^{b}$, Tomas Čižmár ${ }^{c}$ and Andrea Di Falco ${ }^{a}$ \\ ${ }^{a}$ SUPA, University of St Andrews, School of Physics and Astronomy, St Andrews, KY16 9SS, \\ UK ${ }^{b}$ Macquarie University, Department of Physics and Astronomy, Sydney, NSW 2109, \\ Australia ${ }^{c}$ University of Dundee, School of Engineering, Physics and Mathematics, Dundee, \\ DD1 4HN, UK
}

\begin{abstract}
Photonic membranes (PMs) are thin, highly-flexible, membranes which can be imbued with specific photonic functionalities when used to play host to plasmonic features. ${ }^{1-3} \mathrm{PMs}$ can then take that photonic functionality and transfer it to an external object, provided that they can be manipulated with enough precision. We demonstrate a fabrication and optical manipulation protocol that allows PMs to be manoeuvred through a microfluidic environment, and show that the trap stiffness of such a scheme is on par with current techniques. The PMs shown here are $90 \mathrm{~nm}$ thick, with the potential to be extremely flexible. We comment on their current deformability.
\end{abstract}

Keywords: Optical Tweezers, Optomechanics, Photonic Membranes

\section{INTRODUCTION}

Photonic membranes are thin, flexible, and robust membranes that can vary in thickness from tens of nm to several $\mu \mathrm{m}$. In their lateral aspect they are typically made to be between a few $\mu \mathrm{m}$ to several $\mathrm{cm}$ in side length. They are useful in their function as a scaffolding upon which to define nanoplasmonic features. These features can be designed for a particular photonic application, for example filtering, or sensing.

PMs have successfully been used in macroscopic experiments, however, due to the precision with which they must be positioned, they have not yet been deployed in a microfluidic environment. One tool which can provide

Further author information: (Send correspondence to B.C.K)

B.C.K.: E-mail: bck2@st-andrews.ac.uk, Telephone: 01334421605

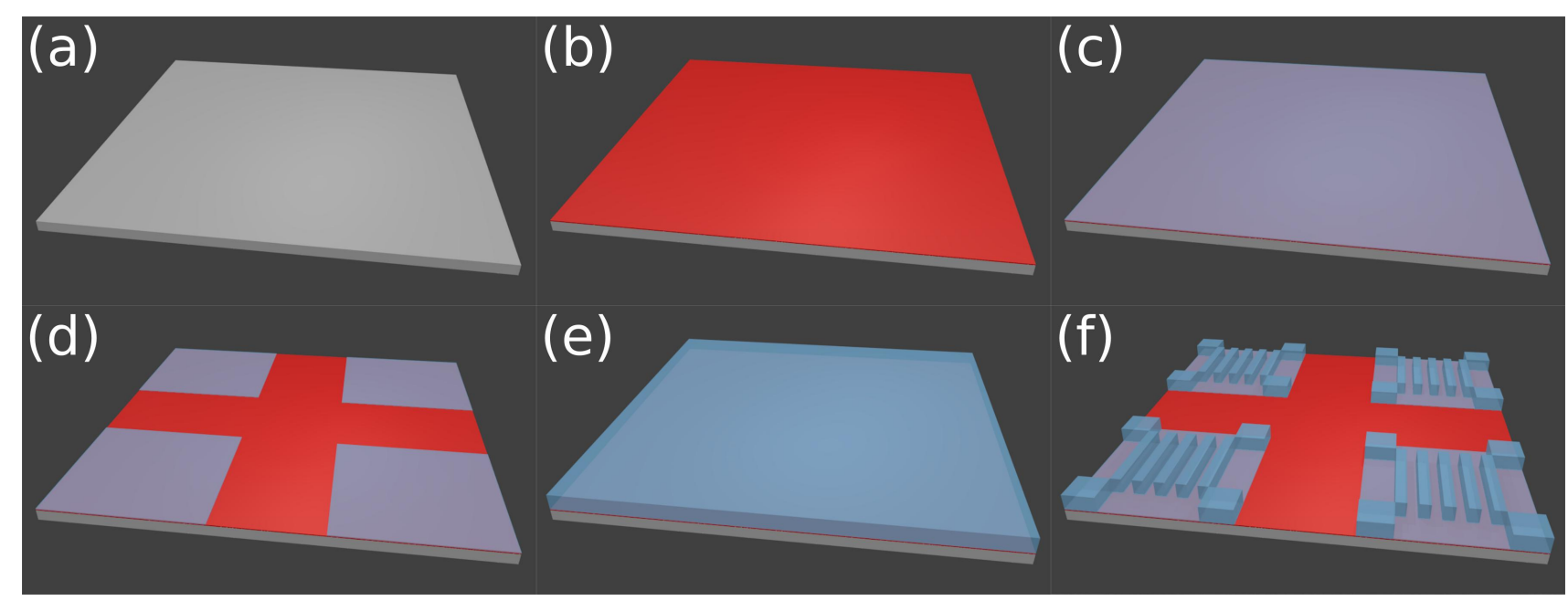

Figure 1. Fabrication procedure for photonic membranes. (a) Fabrication starts with a piece of silicon wafer, (b) onto which a sacrificial layer is spun, followed by (c) a layer of SU-8 photoresist, $40 \mathrm{~nm}$ in thickness. (d) SU-8 is patterned via electron-beam lithography and developed chemically. (e-f) This process is repeated with a second, thicker, layer of SU-8 from which handles are defined. Upon removal of the sacrificial layer, the PMs are left freely suspended in solution.

Optical Trapping and Optical Micromanipulation XIII, edited by Kishan Dholakia, Gabriel C. Spalding, Proc. of SPIE Vol. 9922, 99222A · @ 2016 SPIE · CCC code: 0277-786X/16/\$18 · doi: 10.1117/12.2238822 


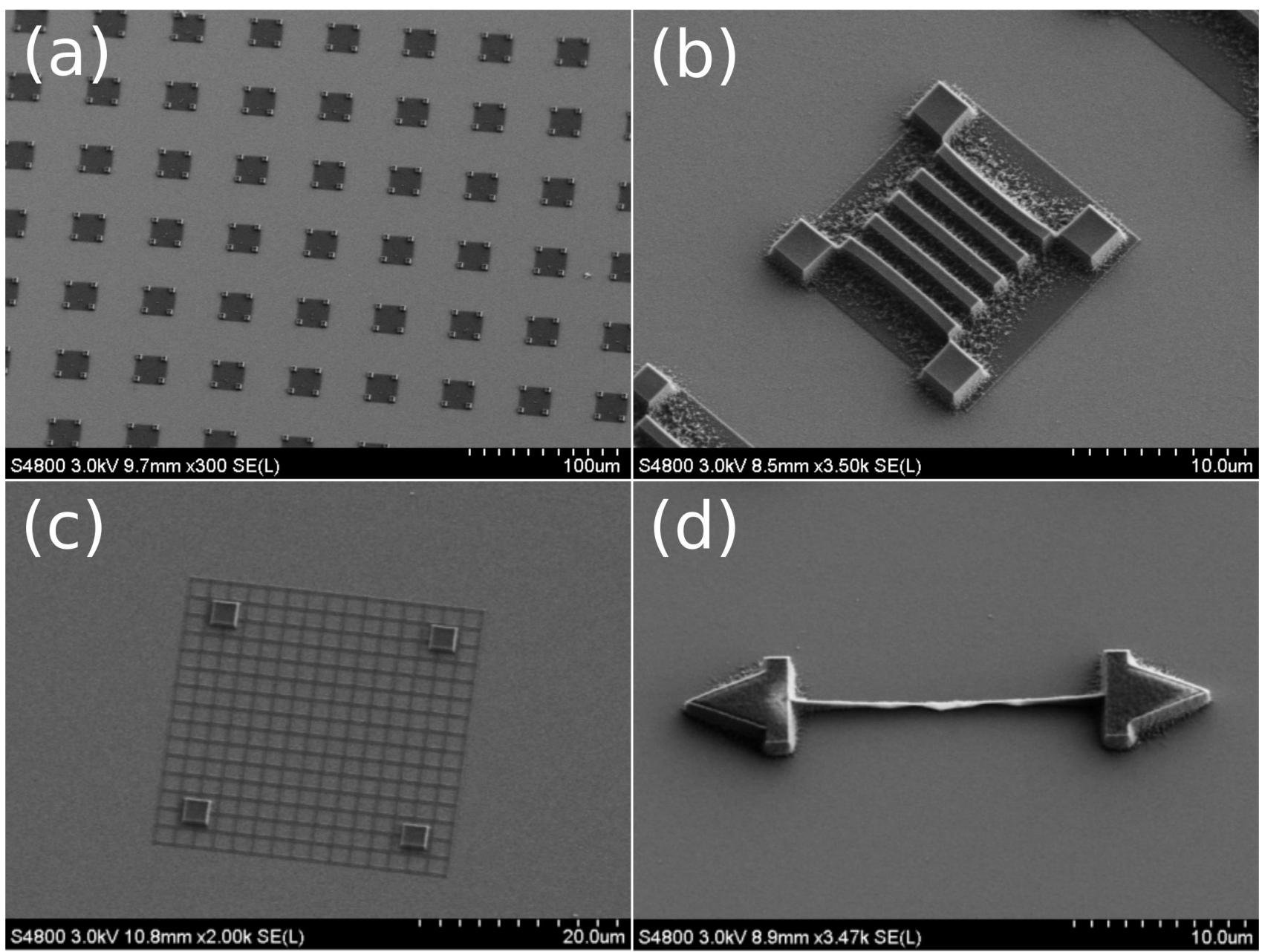

Figure 2. Images of photonic membranes taking using scanning electron microscopy. (a) An array of basic membranes with handles patterned in each corner. (b-c) Close-ups of two particular PMs, with (b) a polymeric grating added to the membrane, and (c) a fishnet membrane, designed to allow for greater membrane flexibility. (d) A microtool structure, also defined from SU-8.

ample control at this scale is optical tweezers, ${ }^{4,5}$ and yet they are historically not compatible with objects that have planar geometries, such as membranes.

By defining our PMs from SU-8 photoresist ${ }^{6}$ using electron beam lithography, we can create PMs with "handles" at each of their four corners. These handles can be trapped via optical tweezers, and by moving them, the entire PM can be manipulated with a high degree of precision.

\section{RESULTS}

\subsection{Fabrication of Photonic Membranes}

The fabrication protocol for PMs begins with a silicon substrate, as shown in figure 1, in order to ensure that the subsequent layers of SU-8 photoresist are uniform. We use a spin-coater to spin a sacrificial layer of Omnicoat on top of the silicon, followed by a layer of SU- 8 that is $40 \mathrm{~nm}$ thick. After being baked at $100^{\circ}$ for 5 minutes, the sample is patterned via electron-beam lithography. SU-8 is a negative-tone photoresist, meaning that areas that are exposed to the e-beam become crosslinked and will remain unaffected by chemical development. Unexposed areas of SU-8 will be dissolved during the chemical development process, leaving only the patterned area. This procedure is repeated a second time, with an additional layer of SU-8 (1.5 $\mu \mathrm{m}$ thick) being spin-coated, baked, 


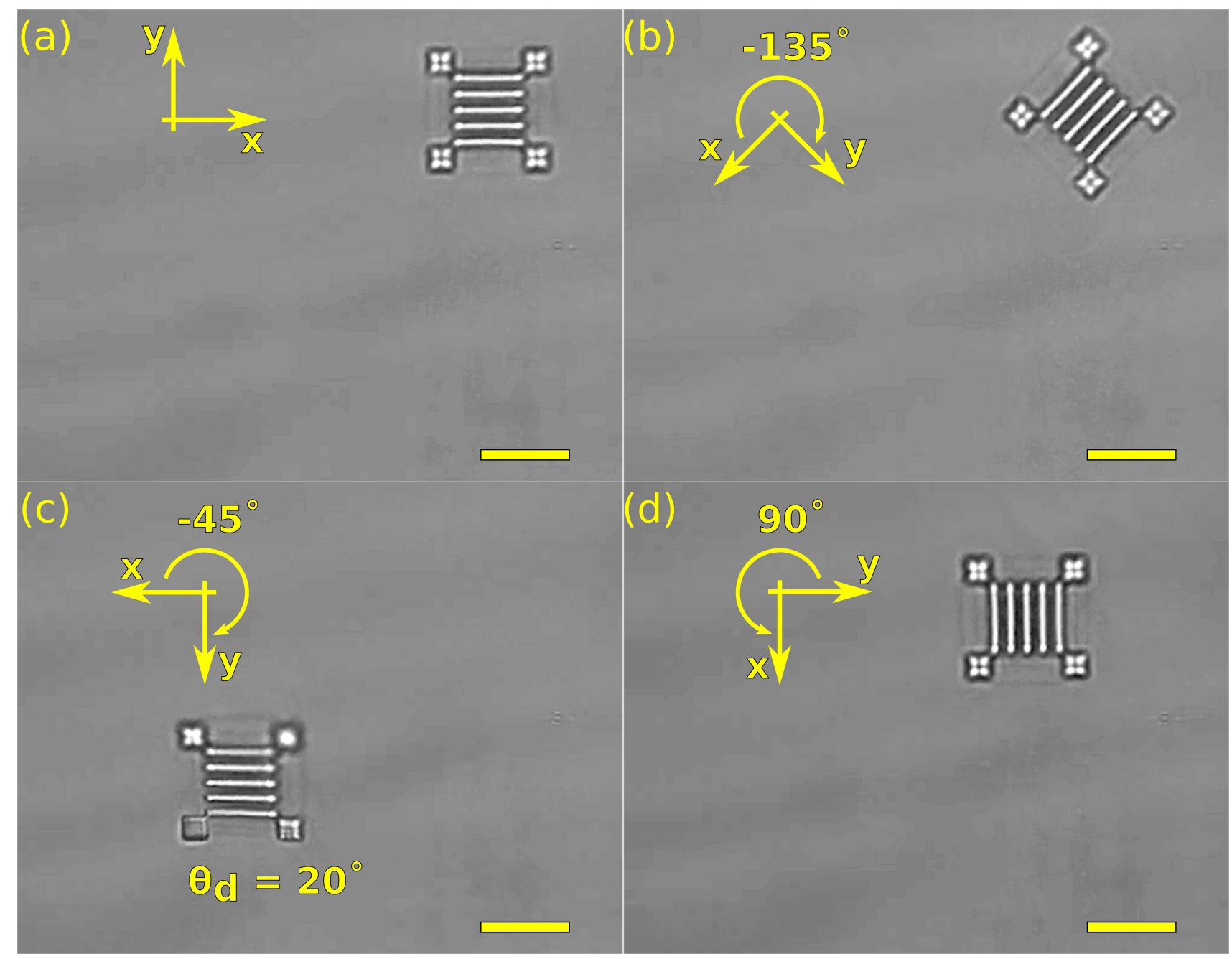

Figure 3. Key frames extracted from video showing the automated movement of membranes. (a) "Starting" position of PM, with grating aligned in a left-right orientation. (b) PM is rotated clockwise through an angle of $135^{\circ}$ before being (c) translated and rotated simultaneously, this time through $45^{\circ}$. It is then rotated out of focal plane by an angle $\theta_{d}=20^{\circ}$. (d) The PM is returned to its "in=plane" orientation $\left(\theta_{d}=0^{\circ}\right)$ and translated back to the upper right region of the field of view, again while being rotated, this time by $90^{\circ}$ in an anti-clockwise direction. X-and y-axes mark the orientation of the membrane in each frame. Yellow scale bars are $20 \mu \mathrm{m}$ in length.

patterned, and developed. It is from this second layer that the handles of the PM are defined, as too are the polymeric grating structures shown in figure 2(b).

With PMs fully realised, it remains only to dissolve the sacrificial layer by immersing the sample in MF319 silicon developer (Microchem) for a few minutes at room temperature. At this stage, one has PMs freely suspended in solution. Microscope slides can be easily prepared using this PM solution.

Many different PMs can be made using this fabrication protocol, a sample of which are shown in figure 2. Figure 2(a) shows an array of basic PMs, consisting simply of a solid membrane with one handle structure in each corner. Variations on the basic design include the addition of a polymeric "grating" which can be useful as a guide to the eye during experiments, and a membrane made in a fishnet design which have a higher degree of flexibility than solid membranes. Additionally, microtools like the one shown in figure 2(d) can be used in a microfluidic setting for particle sorting applications. 


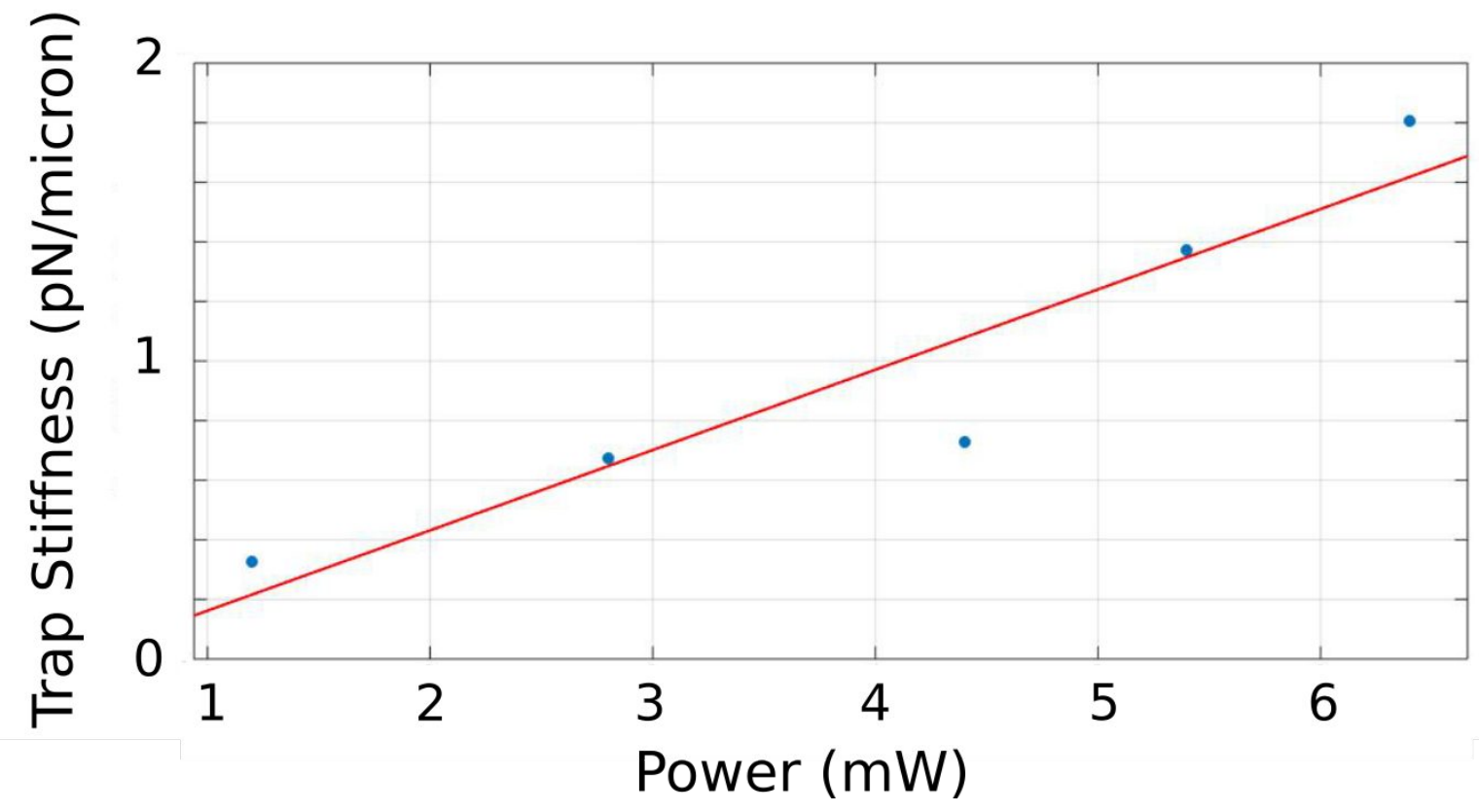

Figure 4. Performance of the optical tweezers system as it relates to the trapping of a PM. Powers given here represent the upper limit on estimated power in each trapping beam, calculated by measuring optical power at back focal plane of the objective lens and then multiplying this by the quoted transmission for objective.

\subsection{All-optical manipulation of Photonic Membranes}

We use a custom-built holographical optical tweezers (HOT) system, ${ }^{7-9}$ operating at a wavelength of $830 \mathrm{~nm}$, to manipulate the photonic membranes. ${ }^{10}$ Traps were generated and controlled via a LabView programme which was based on the commonly used "superposition of gratings and lenses" (SGL) algorithm. ${ }^{11}$

Typically four traps were generated, and placed manually such that there was one trap per handle. Once the traps were in an acceptable arrangement, this arrangement could be addressed as a whole, either manually or by defining an automated sequence of movements which were carried out programatically. Figure 3 shows such an automated movement routine.

In figure 3(a) four traps are positioned, each on a different handle of the PM. This arrangement is then fixed and addressed as a whole. Figure $3(\mathrm{~b}-\mathrm{d})$ shows the PM being rotated through $135^{\circ}$, translated through the microscope cell where it is then tilted out of the focal plane to angle of $\theta_{d}=0^{\circ}$. While in this orientation it is rotated again, this time through an angle of $45^{\circ}$ degrees. Finally, it is returned to a "flat" orientation $\left(\theta_{d}=0^{\circ}\right)$ before being translated and rotated simultaneously.

The trapping of the PMs was found to be very stable, with the membranes capable of being manipulated with a high degree of control. Figure 4 shows a plot of trap stiffness versus power for the trapped PMs. The powers quoted were estimated by first measuring the optical power in the back focal plane of the objective lens, and multiplying this value by the objective lens' quoted transmission.

In addition to having control over the position and orientation of the PMs, we can also deform them. Figure 5(a) shows a PM in its default state. This PM was patterned such that the membrane layer was not solid, but rather it was a fishnet structure. This modification lowered the PM's flexural rigidity which made it easier to bend. Figure 5(b) shows the same PM in a deformed state, where by corners of the membrane were moved by either altering the focus of the trapping beams to be above or below the focal plane of the microscope. Similar deformation can be seen in figure $5(\mathrm{c}-\mathrm{d})$ where a single thread of SU-8 is bent by moving the two handle structures towards each other in a pincer-like motion. 


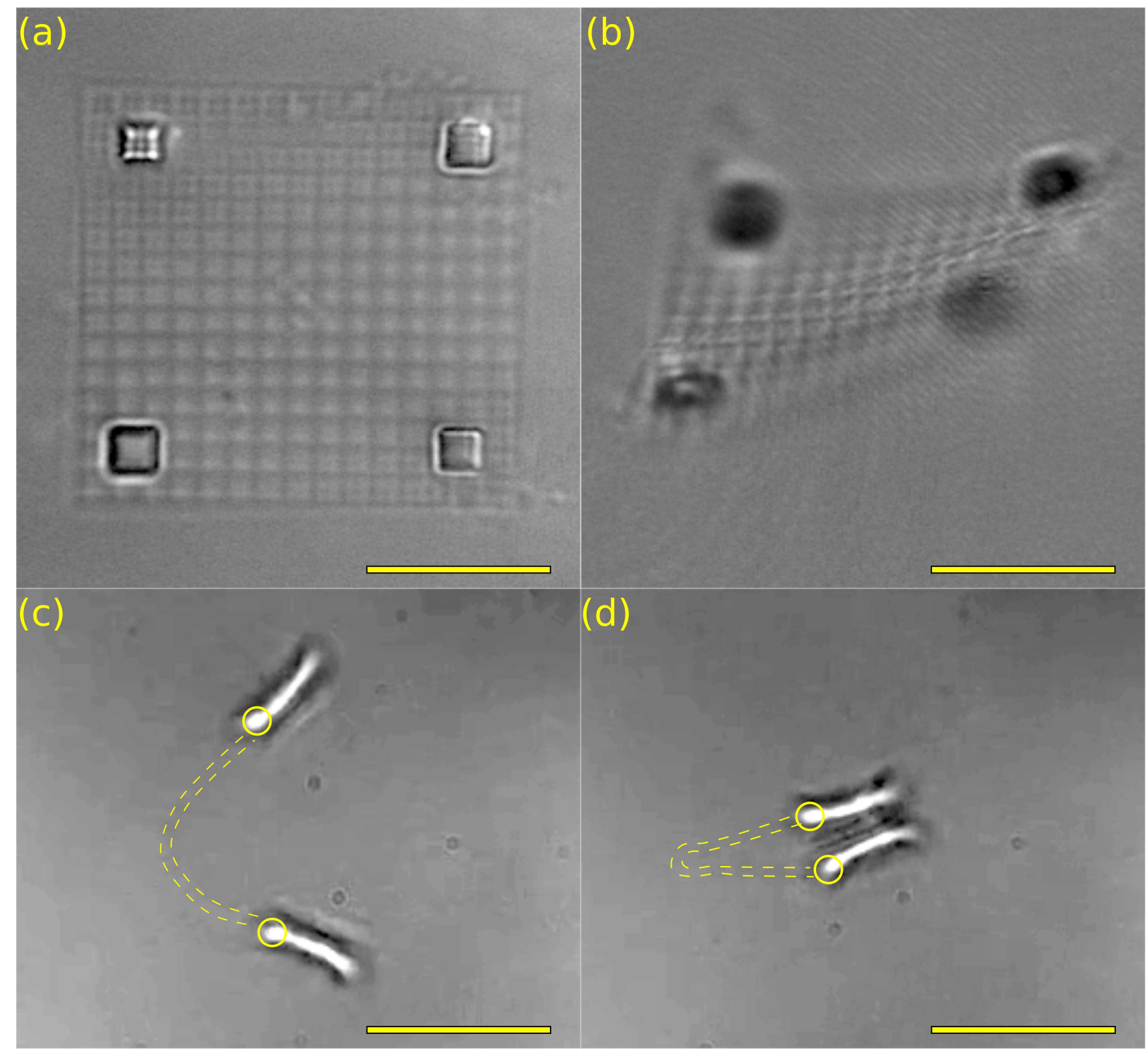

Figure 5. Images highlighting the flexibility of photonic membranes. (a-b) PMs with fishnet structures experience a decrease in their flexural rigidity, allowing them to be deformed from (a) neutral state to (b) a warped state through the modulation of the four trapping beams. (c-d) Similar deformation shown on a single strand of SU-8 in a microtool structure. (c) The device shown is trapped at the location of the yellow rings, and can be readily opened and closed using a pincer-like movement. (d) The extreme case whereby the device is completely "closed" demonstrates the flexibility of SU-8. The dashed lines in the bottom two panels define the boundaries of the SU-8 threads, which are very difficult to see under the microscope. Scale bars are $20 \mu \mathrm{m}$. 


\section{CONCLUSION}

We have shown that PMs are capable of being manipulated with a high degree of precision, both in terms of their position and orientation, through the utilisation of an appropriate fabrication and manipulation protocol. The trap stiffness obtainable with such a scheme is on a par with that found in literature, with the PMs potentially benefitting from their increased mass and the fact that the trapped handles are physically linked to one another.

The fact that we can now manipulate PMs in a microfluidic environment leads us to relevant biological applications. By taking the basic PMs shown here, and using them as a hosts for nanoplasmonic features, we proceed to investigate the deployment of microfluidic sensors and filters capable of taking localised, in-situ, readings of their environments.

\section{REFERENCES}

[1] Falco, A. D., Ploschner, M., and Krauss, T. F., "Flexible metamaterials at visible wavelengths," New Journal of Physics 12, 113006 (Nov. 2010).

[2] Reader-Harris, P., Ricciardi, A., Krauss, T., and Di Falco, A., "Optical guided mode resonance filter on a flexible substrate.," Optics express 21(1), 1002-1007 (2013).

[3] Reader-Harris, P. and Di Falco, A., "Nanoplasmonic filters for hollow core photonic crystal fibers," ACS Photonics 1, 985-989 (10 2014).

[4] Ashkin, A., "Acceleration and trapping of particles by radiation pressure," Phys. Rev. Lett. 24, 156-159 (Jan 1970).

[5] Ashkin, A., Dziedzic, J. M., Bjorkholm, J. E., and Chu, S., "Observation of a single-beam gradient force optical trap for dielectric particles," Optics letters 11(5), 288-290 (1986).

[6] Lorenz, H., Despont, M., Fahrni, N., LaBianca, N., Renaud, P., and Vettiger, P., "SU-8: a low-cost negative resist for MEMS," Journal of Micromechanics and Microengineering 7(3), 121 (1997).

[7] Curtis, J. E., Koss, B. A., and Grier, D. G., "Dynamic holographic optical tweezers," Optics Communications 207(June), 169-175 (2002).

[8] Dholakia, K., Reece, P., and Gu, M., "Optical micromanipulation," Chem. Soc. Rev. 37, 42-55 (2008).

[9] Dufresne, E. R., Spalding, G. C., Dearing, M. T., Sheets, S. a., and Grier, D. G., "Computer-generated holographic optical tweezer arrays," Review of Scientific Instruments 72(3), 1810-1816 (2001).

[10] Martin-Badosa, E., Montes-Usategui, M., Carnicer, a., Andilla, J., Pleguezuelos, E., and Juvells, I., "Design strategies for optimizing holographic optical tweezers setups," J. Opt. A: Pure Appl. Opt. 267, 16 (2007).

[11] Di Leonardo, R., Ianni, F., and Ruocco, G., "Computer generation of optimal holograms for optical trap arrays," Optics Express 15(4), 1913-1922 (2007). 